\title{
Polynomial solutions of differential equations
}

\author{
H Azad*, A Laradji and M T Mustafa
}

* Correspondence:

hassanaz@kfupm.edu.sa Department of Mathematics \& Statistics, King Fahd University of Petroleum \& Minerals, Dhahran, Saudi Arabia

\section{Abstract}

A new approach for investigating polynomial solutions of differential equations is proposed. It is based on elementary linear algebra. Any differential operator of the form $L(y)=\sum_{k=0}^{k=N} a_{k}(x) y^{(k)}$, where $a_{k}$ is a polynomial of degree $\leq k$, over an infinite field $F$ has all eigenvalues in $F$ in the space of polynomials of degree at most $n$, for all $n$. If these eigenvalues are distinct, then there is a unique monic polynomial of degree $n$ which is an eigenfunction of the operator $L$, for every non-negative integer $n$. Specializing to the real field, the potential of the method is illustrated by recovering Bochner's classification of second order ODEs with polynomial coefficients and polynomial solutions, as well as cases missed by him - namely that of Romanovski polynomials, which are of recent interest in theoretical physics, and some Jacobi type polynomials. An important feature of this approach is the simplicity with which the eigenfunctions and their orthogonality and norms can be determined, resulting in significant reduction in computational complexity of such problems.

2000 MSC: 33C45; 34A05; 34A30; 34B24.

\section{Introduction}

Polynomial solutions of differential equations is a classical subject, going back to Routh [1], Bochner [2] and Brenke [3] and it continues to be of interest in applications, as in, e.g., $[4,5]$. The idea we wish to present in this article is to conduct the discussion of differential equations with polynomial coefficients in a linear algebraic context. It is surprising that by such a change of view point, one can add more than what is available in the classical literature and, at the same time, recover classical results efficiently and in a unified manner.

We take this opportunity to correct a common misconception regarding Brenke's contributions in the classification of second-order ODEs that have polynomial solutions [6, p. 508]. He first considers all the second-order ODEs that have a polynomial solution in every degree and only subsequently classifies self-adjoint equations by an argument similar to that given in Section 3. He then returns to the general secondorder equation and, for an inexplicable reason, does not carry through the argument to its logical conclusion and misses some important cases.

In this article, we investigate operators of the form $L(\gamma)=\sum_{k=0}^{N} a_{k}(x) y^{(k)}$, where $a_{k}$ is a polynomial of degree $\leq k$, with coefficients in an infinite field $F$. Clearly, any linear $n$ th-order differential operator, which has polynomial coefficients and eigenpolynomials of degrees 0 up to $n$, must be of this form, and, as shown in Section 2, such operators may not have eigenpolynomials in every degree. We show that these operators,

(c) 2011 Azad et al; licensee Springer. This is an Open Access article distributed under the terms of the Creative Commons Attribution License (http://creativecommons.org/licenses/by/2.0), which permits unrestricted use, distribution, and reproduction in any medium, provided the original work is properly cited. 
operating on polynomials, have all their eigenvalues in the field and in case the eigenvalues are distinct, there is exactly one monic polynomial in every degree which is an eigenfunction of $L$.

Specializing to second-order equations because of their importance in applicationsand leaving in this article the higher-order case because of its technical complexity-the canonical forms of second-order equations, their eigenvalues, and multiplicities are investigated. This includes the family of Romanovski polynomials and some Jacobitype polynomials, which are missing in the classification of Brenke and Bochner as well as in the latest books on the subject; the Romanovski polynomials are the main subject of some recent physics literature [5,7].

Necessary and sufficient conditions for a second-order operator to be self-adjoint are obtained and a reduction formula for computations of norms of eigenfunctions of these operators is also given, which avoids the customary case-by-case analysis found, e.g., in $[6,8,9]$.

A complete classification of second-order operators which are self-adjoint with respect to some weight function is also given: among all the polynomial solutions of differential equations, the classical polynomials make their appearance as soon as one searches for self-adjoint operators. This classification is due originally to Brenke [3].

Although one normally assumes that the leading polynomial coefficient of a differential equation should never vanish, it is worth noting that it is precisely the singularities of the equation that encapsulate all the important information about the equation.

In the last section, the important examples of Jacobi and some non-standard polynomials are given in detail.

Regarding eigenvalues we will use the following terminology.

Let $\lambda$ be an eigenvalue of an operator $L$ on a finite dimensional space. The geometric multiplicity of $\lambda$ is the dimension of its eigenspace while its algebraic multiplicity (or multiplicity for short) is its multiplicity as a zero of the characteristic polynomial. Moreover, $\lambda$ is said to be simple if its algebraic multiplicity is equal to 1 , semisimple if its algebraic and geometric multiplicities coincide, and defective if its algebraic multiplicity is greater than its geometric multiplicity.

\section{Basic results}

In this article, $\mathbb{P}$ is the space of all polynomials over an infinite field $F$ and $\mathbb{P}_{n}$ is the subspace of polynomials with degree at most $n$, and for a fixed positive integer $N, L: \mathbb{P}$ $\rightarrow \mathbb{P}$ is the Nth-order operator given by $L y=\sum_{k=1}^{N} a_{k}(x) D^{k} y$, where $D$ is the usual differential operator and $a_{k}(x)$ is a polynomial of degree at most $k(1 \leq k \leq N)$. In this way, for each non-negative integer $n, \mathbb{P}_{n}$ is $L$-invariant. Put $a_{k}(x)=\sum_{h \geq 0} a_{k h} x^{h}$, where $a_{k h}=0$ if $k<h$. As $L\left(x^{j}\right)$ is a scalar multiple of $x^{j}$ plus lower-order terms $(1 \leq j \leq n)$, we see that the matrix representation of $L$, with respect to the standard basis $B_{n}=\{1$, $\left.x, \ldots, x^{n}\right\}$, is upper triangular and the eigenvalues are the coefficients of $x^{j}$ in $L\left(x^{j}\right)(1 \leq j$ $\leq n)$. In more detail, the $(n+1) \times(n+1)$ matrix of $L$ operating on $\mathbb{P}_{n}$ is

$$
A_{n}=\left[\sum_{k \geq 1}(j-k)_{k} a_{k, k+i-j}\right]_{1 \leq i, j \leq n+1}
$$


where $(j-k)_{k}=(j-1)(j-2) \ldots(j-k)$ and $a_{k h}=0$ when $k<h$, so $A_{n}$ is upper triangular (and where each row and each column has at most $N+1$ non-zero entries). Clearly, $A_{n+1}$ is obtained from $A_{n}$ by adding one row and one column at the end, and so all the eigenvalues of the operator $L$ are in $F$ and are given by

$$
\lambda_{0}=0, \lambda_{n}=n a_{11}+n(n-1) a_{22}+\cdots+n ! a_{n n} \text { for } n \geq 1
$$

where $a_{n n}=0$ if $n>N$. Each $\lambda_{n}$ has, as an eigenfunction, a polynomial $y_{n}(x)=y_{n 0}+$ $y_{n 1} x+\ldots+y_{n n} x^{n}$ whose vector representation $\left(y_{n 0}, \ldots, y_{n n}\right)^{T}$ in the standard basis $B_{n}$ of $\mathbb{P}_{n}$ can directly be computed using the homogeneous triangular system

$$
\left(A_{n}-\lambda_{n} I\right)\left(y_{n 0}, \ldots, y_{n n}\right)^{T}=0
$$

In particular, if the eigenvalues $\lambda_{0}, \lambda_{1}, \ldots, \lambda_{n}$ (for some $n$ ) are distinct, then $\mathbb{P}_{n}$ has a basis of eigenfunctions and, for reasons of degree, $L$ has (up to a constant) a unique polynomial of degree $r$ (for each $r, 0 \leq r \leq n$ ) corresponding to $\lambda_{r}$ as an eigenfunction. We summarize this in

Proposition 2.1 Let $L: \mathbb{P} \rightarrow \mathbb{P}$ be an operator given by $L y=\sum_{k=1}^{N} a_{k}(x) D^{k} y$, where $a_{k}(x)$ is a polynomial of degree at most $k$. For each $k(1 \leq k \leq N)$, let $c_{k}$ be the coefficient of $x^{k}$ in $a_{k}(x)$. Then all the eigenvalues of $L$ are in the field $F$ and are $\mathbb{Z}$-linear combinations of the $c_{k}$. If all the eigenvalues are distinct, then $L$ has, up to a constant, a unique polynomial for each degree as an eigenfunction.

Some observations concerning the eigenvalues and their multiplicity are in order. First, let

$$
f(x)=c_{1} x+c_{2} x(x-1)+\cdots+c_{N} x(x-1) \cdots(x-N+1)
$$

where, as in Proposition 2.1, $c_{k}=a_{k k}$ is the coefficient of $x^{k}$ in $a_{k}(x)$. Then each eigenvalue $\lambda_{n}$ of $L$ is just $f(n)(n \geq 0)$. This immediately gives an $(N+1)$-term recurrence relation between the eigenvalues, for if $E$ is the shift operator given by $E f(x)=f(x$ +1 ), then $(E-1)^{N}{ }_{+} f(n)=0$. When all the $c_{k}$ are zero (i.e., all eigenvalues are equal to zero), then $f$ is identically zero and one can get the eigenfunctions of $L$ by considering the $(N-1)$-order operator obtained from $L$ by replacing $y$ by $D y$. We therefore assume that $f$ is not the zero polynomial. Suppose that an eigenvalue is repeated $r$ times, say

$$
\lambda_{n_{1}}=\lambda_{n_{2}}=\cdots=\lambda_{n_{r}} \text {, where } 0 \leq n_{1}<n_{2}<\cdots<n_{r} .
$$

In this case, $f$ takes on the same value at $r$ different non-negative integers, and so $r \leq$ $\operatorname{deg}(f) \leq N$. Moreover, if the field $F=\mathbb{R}$, it is clear that there is a positive integer $u$ such that for each integer $v \geq u$, the set $\{n: f(n)=v\}$ is a singleton, meaning that only finitely many eigenvalues $\lambda_{n}$ of $L$ have multiplicity greater than 1 , and, if any exist, they must all lie between the largest local maximum and the smallest local minimum of $f$.

An interesting fact occurs when $N=2$. Suppose, as before, that not both coefficients $c_{1}$ and $c_{2}$ are zero and that an eigenvalue has algebraic multiplicity 2 , say $\lambda_{n}=\lambda_{n^{\prime}}$ for some non-negative integers $n<n^{\prime}$. Then, from Equation 2.1, $n c_{1}+n(n-1) c_{2}=n^{\prime} c_{1}+$ $n^{\prime}\left(n^{\prime}-1\right) c_{2}$, and, $\lambda_{n+n^{\prime}}=\left(n+n^{\prime}\right) c_{1}+\left(n+n^{\prime}\right)\left(n+n^{\prime}-1\right) c_{2}=0$. Since the multiplicity of the eigenvalue zero cannot exceed 2 , we obtain that for each integer $k>n+n^{\prime}$, the eigenvalue $\lambda_{k}$ has multiplicity 1 . We also see that if $n_{1}+n_{2}=n+n^{\prime}$, where $n_{1}<n_{2}$, 
then $\lambda_{n 1}=\lambda_{n 2}$, meaning that the number of eigenvalues that have multiplicity 2 is $\left\lceil\frac{n+n^{\prime}}{2}\right\rceil$. We thus have

Proposition 2.2 Let the field be $\mathbb{R}$. Then, with the above notation, either all eigenvalues of the $N$ th order operator $L$ are equal to 0 or all have multiplicity 1 except for finitely many of them which will then have multiplicity at most $N$. In case $N=2$, there will be eigenvalues with multiplicity 2 precisely when a non-negative integer $k$ exists for which $c_{1}+k c_{2}=0$, and then the number of such eigenvalues is $\left\lceil\frac{k+1}{2}\right\rceil$.

It is perhaps tempting to think that although the eigenvalues of the operator $L$ may not be distinct, yet it is still diagonalizable. As Proposition 2.3 shows, this is not always the case.

We now concentrate on second-order operators. Let $L(y)=a(x) y^{\prime \prime}+b(x) y^{\prime}$, where $\operatorname{deg}(a)=2, \operatorname{deg}(b) \leq 1$. Following Bochner [2], by scaling and translation, we may assume that $a(x)=x^{2}-1, x^{2}+1$ or $x^{2}$. We then have the following result.

Proposition 2.3 (i) The equation $\left(x_{2}+\varepsilon\right) y^{\prime \prime}+(\alpha x+\beta) y^{\prime}+\lambda y=0, \varepsilon=0,1,-1$ has unique monic polynomial solutions in every degree if $\alpha>0$ or if $\alpha<0$ and it is not an integer.

If $\alpha=-(n+m-1)$ for $0 \leq m \leq(n-1)$, then the eigenvalue $\lambda=n(n-1)+\alpha n=$ $-n m$ has algebraic multiplicity 2 and eigenpolynomials can only be of degree $n$ or $m$. An eigenpolynomial $y=\sum_{k=0}^{n} a_{k} x^{k}$ is of degree $n$ if and only if

$$
\varepsilon a_{m+2}(m+2)(m+1)+\beta a_{m+1}(m+1)=0
$$

in which case the $\lambda$ eigenspace in $\mathbb{P}_{n}$ is two-dimensional; otherwise the $\lambda$ eigenspace is one-dimensional.

(ii) The equation $x y^{\prime \prime}+(\alpha x+\beta) y^{\prime}+\lambda y=0$ has unique monic polynomial solutions in every degree if $\alpha \neq 0$.

(iii) The equation $y^{\prime \prime}+(\alpha x+\beta) y^{\prime}+\lambda y=0$ has unique monic polynomial solutions in every degree if $\alpha \neq 0$.

\section{Proof.}

(i) Let $L(y)=\left(x^{2}+\varepsilon\right) y^{\prime \prime}+(\alpha x+\beta) y^{\prime}$, where $\varepsilon=0,1,-1$. By Proposition 2.1 or noticing that the eigenvalues are given by the coefficients of $x^{n}$ in $L\left(x^{n}\right)$, these eigenvalues are $\lambda=n(n-1)+\alpha n$. Suppose such an eigenvalue is a repeated eigenvalue. Then, $L$ $\left(x^{m}\right)=\lambda x^{m}+$ lower degree terms, where $m \neq n$. Therefore, $n(n-1)+\alpha n=m(m-1)$ $+\alpha m$ which gives $\alpha=-(n+m-1)$. This means that if $\alpha$ is not an integer or if $\alpha$ is a positive integer, then the operator $L$ has distinct eigenvalues. In this case, there is up to a scalar only one polynomial in every degree which is an eigenfunction of $L$.

Now suppose $\alpha=-(n+m-1)$ for distinct non-negative integers $n, m$ and $\lambda=n(n$ $-1)+\alpha n=-n m$. We may assume that $n>m$. Suppose $L\left(x^{k}\right)=\lambda x^{k}+$ lower degree terms, with $k \neq n$. Then $\alpha=-(n+m-1)=-(n+k-1)$ gives $k=m$. Therefore, if there is a repeated eigenvalue, it is of multiplicity 2 and eigenpolynomials can only be of degrees $m$ and $n$. Moreover, if $\alpha=-(n+m-1)=-(i+j-1)$ then the eigenvalue $-i j$ is also repeated. 
Since the algebraic multiplicity of an eigenvalue is always greater than or equal to its geometric multiplicity, a non-repeated eigenvalue of $L$ has exactly one monic polynomial as an eigenfunction.

Let us now determine the geometric multiplicities of all the eigenvalues in case $\alpha$ is a non-positive integer.

Let $\alpha=-(n+m-1)$ where $n>m \geq 0$. As seen above the eigenvalue $\lambda=n(n-1)+$ $\alpha n=-n m$ is of algebraic multiplicity 2 and the corresponding eigenpolynomials can only be in degrees $n$ and $m$. If $y=\sum_{k=0}^{n} a_{k} x^{k}$ then

$$
\begin{aligned}
L(y)= & \sum_{k=0}^{n-2}\left[a_{k} k(k-1)+\varepsilon a_{k+2}(k+2)(k+1)+\alpha a_{k} k+\beta a_{k+1}(k+1)\right] x^{k} \\
& +\left[a_{n-1}(n-1)(n-2)+\alpha a_{n-1}(n-1)+\beta a_{n} n\right] x^{n-1}+[n(n-1)+\alpha n] a_{n} x^{n}
\end{aligned}
$$

The solutions of $L(y)=\lambda y=(n(n-1)+\alpha n) y=-(n m) y$ satisfy therefore

$$
a_{k} k(k-1)+\varepsilon a_{k+2}(k+2)(k+1)+\alpha a_{k} k+\beta a_{k+1}(k+1)=\lambda a_{k \prime} \quad(k=0, \ldots, n)(2.2)
$$

where $a_{n+1}=0, a_{n+2}=0$ and $a_{n} \neq 0$. From Equation 2.2 we can solve for $a_{k}$ in terms of $a_{k+1}, a_{k+2}$ provided $(k(k-1)+\alpha k-\lambda) \neq 0$. Therefore, we can solve for all $a_{k}$ with $k$ $>m$ in terms of $a_{n}$. Equation 2.2 for $k=m$ reads

$$
\varepsilon a_{m+2}(m+2)(m+1)+\beta a_{m+1}(m+1)=[\lambda-m(m-1)-\alpha m] a_{m}=0 .
$$

If Equation 2.3 holds then $a_{m}$ can be arbitrary and every $a_{k}$ for $k<m$ is determined in terms of $a_{m}$ and $a_{n}$. In this case, the $\lambda$ eigenspace is two-dimensional. If Equation 2.3 does not hold then there is no eigenpolynomial of degree $n$. In this case, there will be a unique monic polynomial of degree $m$.

(ii \& iii) Here the proofs follow from the fact that the eigenvalues of the operator $L$ in both the cases are $\alpha n$ so, for $\alpha \neq 0$, all the eigenvalues are distinct.

Proposition 2.3 shows, in particular, that for Jacobi-type differential equations, there are cases where the algebraic and geometric multiplicities are equal to 2 and cases where the algebraic multiplicity is 2 and the geometric multiplicity is 1 (Cf. [2]).

Corollary 2.4 Let $L(y)=x^{2} y^{\prime \prime}+(\alpha x+\beta) y^{\prime}$.

(i) If $\alpha$ is not a non-positive integer then all the eigenvalues of $L$ are simple.

(ii) If $\alpha=-(n+m-1)$ where $n>m \geq 0$ then all eigenvalues $\lambda$ except $\lambda=-n m$, are simple and the eigenvalue $-n m$ has multiplicity 2 .

In this case if $\beta=0$ then all the eigenvalues are semisimple with eigenpolynomials ${ }^{k}$ $(k=0,1, \ldots)$.

If $\beta \neq 0$ then the repeated eigenvalue $-n m$ is defective with eigenpolynomial

$$
\sum_{l=0}^{m}(-\beta)^{m}\left(\begin{array}{c}
m \\
l
\end{array}\right) \frac{(n-m) !}{(n-l) !}\left(-\frac{x}{\beta}\right)^{l}
$$

Proof. (i) and the first statement of (ii) have already been proved in Proposition 2.3. Using Equation 2.3 in the proof of Proposition 2.3 with

$$
\alpha=-(n+m-1) \quad 0 \leq m \leq n-1
$$


and

$$
\lambda=n(n-1)+\alpha n=-n m
$$

we get

$$
(n-k)(k-m) a_{k}=\beta a_{k+1}(k+1)+\varepsilon a_{k+2}(k+2)(k+1) .
$$

Therefore, for $\varepsilon=0$ we get

$$
(n-k)(k-m) a_{k}=\beta a_{k+1}(k+1)
$$

- If $\beta=0$, then $(n-k)(k-m) a_{k}=0$. Therefore, all $a_{k}$ are zero except for $k=n$ and $k=m$. The general solution is therefore a linear combination of $x^{n}$ and $x^{m}$. The eigenvalue $-n m$ in this case is of geometric multiplicity 2 .

- Assume $\beta \neq 0$. For $k=n$ and $k=m$ Equation 2.4 gives $0=\beta a_{n+1}(n+1)$ and $0=$ $\beta a_{m+1}(m+1)$. Since $a_{n+1}$ is zero, if there is an eigenpolynomial of degree $n$ then we must have $a_{m+1}=0$. This means that there cannot be an eigenpolynomial of degree $n$.

Now from Equation 2.4 we get

$$
a_{k}=\frac{\beta a_{k+1}(k+1)}{(n-k)(k-m)} 0 \leq k \leq m-1
$$

Taking $a_{m}=1$ we get

$$
a_{k-1}=\frac{-\beta a_{k} k}{(n-(k-1))(m-(k-1))} 1 \leq k \leq m
$$

This gives recursively

$$
a_{m-k}=(-1)^{k} \beta^{k}\left(\begin{array}{c}
m \\
k
\end{array}\right) \frac{(n-m) !}{(n-m+k) !}
$$

Substituting $m-k=l$ we get

$$
a_{l}=(-1)^{m-l} \beta^{m-l}\left(\begin{array}{c}
m \\
l
\end{array}\right) \frac{(n-m) !}{(n-l) !}
$$

which gives the monic eigenpolynomial of degree $l$ given by

$$
\sum_{l=0}^{m}(-\beta)^{m}\left(\begin{array}{c}
m \\
l
\end{array}\right) \frac{(n-m) !}{(n-l) !}\left(-\frac{x}{\beta}\right)^{l}
$$

In Proposition 2.3, there is no claim to any kind of orthogonality properties. Nevertheless, the non-classical functions appearing here are of great interest in Physics and their properties and applications are investigated in $[5,7,10]$.

Now the equations $a(x) y^{\prime \prime}+b(x) y^{\prime}+c(x) y=\lambda x$ can be written as second-order Sturm-Liouville equations in the sense of [9, p. 291] by multiplying by a suitable weight function [8, p. 45] and for suitable boundary conditions. A natural question is: What is the explanation for the weight function and the particular form of the boundary conditions?

The following proposition shows that both the weight and general boundary conditions are forced upon us as soon as we demand that the operator $L(y)=a(x) y^{\prime \prime}+b(x) y$ $'+c(x) y$ should be self-adjoint for some weight function $p$. The weight function can be 
determined from the following well-known proposition whose proof can be found for example in [3].

Proposition 2.5 Let $L$ be the operator defined by $L y=a(x) y^{\prime \prime}+b(x) y^{\prime}+c(x) y$ on $a$ linear space $C$ of functions which are at least twice differentiable on a finite interval $I$. Define a bilinear function on $C$ by $(y, u)=\int_{I} p y u d x$, where $p \in C$ is non-negative and does not vanish identically in any open subinterval of I. Then

$(L y, u)-(y, L u)=\left.p a\left(u y^{\prime}-u^{\prime} y\right)\right|_{I}$ for all $y, u \in C$ if and only if $(p a)^{\prime}=p b$

\subsection{Norms of eigenfunctions}

The norms of the eigenfunctions relative to the weight $p$ can be obtained using the well-known three-term recurrence relation for orthogonal polynomials. We include a proof for the readers' convenience and because it is the main point in computation of norms of eigenfunctions.

Proposition 2.6 [8, p. 306] If $\left\{P_{n}\right\}_{n=0,1,2} \ldots$ is a sequence of orthogonal polynomials, then in the expression

$$
x P_{n}=\sum_{j=0}^{j=n+1} k_{j} P_{j},
$$

all the coefficients are 0 except for $j=n+1, n, n-1$.

Proof. Denoting the inner product by round brackets, we have

$$
k_{j}\left(P_{j}, P_{j}\right)=\left(x P_{n}, P_{j}\right)=\left(P_{n}, x P_{j}\right)=0,
$$

if $j+1 \leq n-1$ that is, if $j \leq n-2$, which is what we wanted to show.

Now

$$
x P_{n}=k_{n+1} P_{n+1}+k_{n} P_{n}+k_{n-1} P_{n-1}
$$

Let us rewrite this equation as

$$
x P_{n}=a_{n} P_{n+1}+b_{n} P_{n}+c_{n} P_{n-1}
$$

As there is only one monic eigenpolynomial in every degree, the differential equation must determine all the coefficients. We assume that all eigenfunctions are normalized to be monic.

So $P_{n}=x P_{n-1}+q(x)$, where $\operatorname{deg}(q) \leq n-1$. Therefore $\left(P_{n}, P_{n}\right)=\left(x P_{n-1}, P_{n}\right)=\left(P_{n-1}\right.$, $\left.x P_{n}\right)=\left(P_{n-1}, c_{n} P_{n-1}\right)=c_{n}\left(P_{n-1}, P_{n-1}\right)$-using Equation 2.4 and orthogonality of eigenfunctions of different degrees.

Now, from the differential equation, determining the leading three coefficients of every $P_{n}$ and using Equation 2.4 leads to the determination of $c_{n}$, taking into account that $a_{n}=1$. This gives $\left(P_{n}, P_{n}\right)=c_{n} c_{n-1} \ldots c_{1}\left(P_{0}, P_{0}\right)$ and $\left(P_{0}, P_{0}\right)$ is the integral of the weight function $p$ over an appropriate interval.

The values of $a_{n}, b_{n}, c_{n}$ for classical orthogonal polynomials are given in the table below. 
Table 1 Leading Terms for Classical Polynomials

\begin{tabular}{llll}
\hline Polynomial & $\boldsymbol{a}_{\boldsymbol{n}}$ & $\boldsymbol{b}_{\boldsymbol{n}}$ & $\boldsymbol{c}_{\boldsymbol{n}}$ \\
\hline Legendre & 1 & 0 & $\frac{n^{2}}{(2 n+1)(2 n-1)}$ \\
Hermite & 1 & 0 & $\frac{n}{2}$ \\
Laguerre & 1 & $2 n+1$ & $n^{2}$ \\
Chebychev & 1 & 0 & $\frac{1}{4}($ for $n \geq 2)$ \\
Jacobi & 1 & $\frac{-\beta(2+\alpha)}{(2 n-2-\alpha)(2 n-\alpha)}$ & $\frac{n(n-\alpha-2)(2 n-(\beta+\alpha+2))(2 n+(\beta-\alpha-2))}{(2 n-\alpha-3)(2 n-2-\alpha)^{2}(2 n-\alpha-1)}($ for $n \geq 2)$ \\
& & $b_{1}=\frac{\beta(2+\alpha)}{\alpha(2-\alpha)}$ & $\begin{array}{l}c_{1}=\frac{(\alpha-\beta)(\alpha+\beta)}{(1-\alpha) \alpha^{2}} \\
\end{array}$ \\
& & & $:$ here $\alpha<\beta<-\alpha$ \\
\hline
\end{tabular}

\section{Canonical forms of self-adjoint second-order equations with polynomial coefficients}

Let us now determine the operators for which there is a basis of orthogonal eigenpolynomials for the weight function determined by the operator. The results of this section were arrived at independently; however, the authors found later that such a classification was done first by Brenke [3].

From Proposition 2.5, the operator $L$ would be self-adjoint if there is no contribution from the boundary terms: this is ensured if the product $a(x) p(x)$ vanishes at the end points of the interval-finite or infinite-on which the natural weight function $p(x)$ associated to $L$ is integrable on the entire interval.

The integrability of the weight function determines the differential equation and finiteness of the norm of polynomials ensures that manipulations as in Proposition 2.5 are legitimate. The operator $L$ will then be self-adjoint and it will operate on the vector space of all polynomials of degree at most $n$ for every non-negative integer $n$.

As $L$ has a basis of eigenvectors in any finite dimensional subspace on which it operates, we see that there will be monic polynomial of degree $n$, which will be an eigenfunction of $L$, and the corresponding eigenvalues would therefore be determined from the form of the equation. If these eigenvalues are distinct for different degrees, these polynomials would automatically be orthogonal.

The operator $L$ can be determined from the following requirements

(1) the leading term $a(x)$ is non-zero and of degree at most 2 , the degree of $b(x)$ is at most 1 and $c(x)$ is a constant;

(2) the natural weight function associated to $L$ is integrable on the interval $I$ determined by roots of $a(x)$;

(3) $a(x) p(x)$ vanishes at the end points of $I$ and, in case there is an end point at infinity, the product $a(x) p(x) P(x)$ should vanish at infinity for all polynomials $P(x)$;

(4) all polynomials should have finite norm on the interval $I$ with the weight $p(x)$.

Case I. The polynomial $a(x)$ has two distinct real roots.

By a linear change of variables and scaling, we may assume that the roots are 1 and -1 . Assuming that $a(x)$ is non-negative in the interval $[-1,1]$, we have $a(x)=1-x^{2}$. 
Let $b(x)=\alpha x+\beta$ so

$$
\frac{b(x)}{a(x)}=\frac{\alpha x+\beta}{(1-x)(1+x)}=\frac{\frac{\beta+\alpha}{2}}{1-x}+\frac{\frac{\beta-\alpha}{2}}{1+x} .
$$

So the weight $p(x)$ is

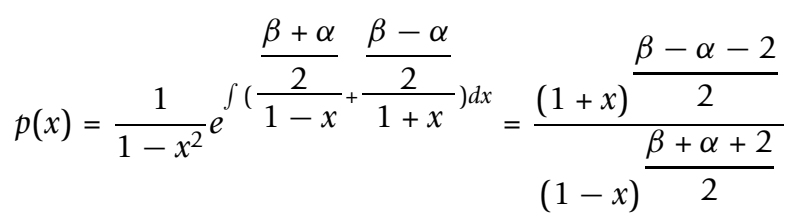

The weight is obviously finite in the interval $(-1,1)$. For $p(x)$ to be integrable we must have $\beta-\alpha>0$ and $\beta+\alpha<0$. Thus, $\alpha<\beta<-\alpha$, so $\alpha<0$.

Case II: The polynomial $a(x)$ has repeated real roots. In this case, we can assume that $a(x)=x^{2}$. Let $b(x)=\alpha x+\beta$. The weight function is now $p(x)=\frac{1}{x^{2}} e^{\int \frac{\alpha x+\beta}{x^{2}} d x}=\frac{|x|^{\alpha}}{x^{2}} e^{-\beta / x}$. We may take the interval $I=(0, \infty)$.

In this case a necessary condition for the integrability of the weight is that $\operatorname{deg}(b)$ $-\operatorname{deg}(a)+1>0$, so this case does not arise.

Case III: The polynomial $a(x)$ is linear.

In this case, we can take $a(x)=x$. Let $b(x)=\alpha x+\beta$. In this case the weight function is $p(x)=\frac{1}{|x|} e^{\int \frac{\alpha x+\beta}{x} d x}=|x|^{\beta-1} e^{\alpha x}$. This is integrable near zero if and only if $\beta \geq 1$. Since $\int_{0}^{\infty} e^{\alpha x} x^{\varepsilon} d x$, where $\varepsilon>0$, is finite only if $\alpha \leq 0$ we see that we cannot take the interval $I$ from $-\infty$ to $\infty$. Without loss of generality we can take this to be the interval $[0, \infty)$. So, the weight function is now $p(x)=x^{\beta-1} e^{\alpha x}$ with $\alpha<0$ and $\beta \geq 1$. All polynomials have finite norm with respect to this weight and for all polynomials $p(x)$ the product $P(x) p(x)$ vanishes at 0 and $\infty$. Therefore, the equation $x y^{\prime \prime}+(\alpha x+\beta) y^{\prime}+\lambda y=0$ has polynomial solutions for every degree $n$. The corresponding eigenvalue is $\lambda=-\alpha n$.

Case IV: $\alpha(x)=1$

In this case $L(y)=y^{\prime \prime}+(\alpha x+\beta) y^{\prime}+\gamma y$. The weight is $p(x)=e^{\frac{\alpha x^{2}}{2}} e^{\beta x}$. So $\alpha$ must be negative, for the product $P(x) p(x)$ to vanish at the end points of the interval $I$ for all polynomials $P(x)$, and therefore $I$ must be $(-\infty, \infty)$.

Remark The case of a second degree $a(x)$ with no real roots does not arise, because of the requirements (3) and (4) above which a weight function must satisfy.

\section{Examples}

As illustration of the ideas of previous sections, we discuss the Jacobi polynomials and two non-standard examples including the Romanovski polynomials.

\subsection{Jacobi polynomials}

First note that for any differentiable function $f$ with $f^{\prime}$ continuous, the integral $\int_{0}^{\varepsilon} \frac{f(x)}{x^{\alpha}} d x$ is finite if $\alpha<1-$ as one sees using integration by parts. 
Consider the equation $\left(1-x^{2}\right) y^{\prime \prime}+(\alpha x+\beta) y^{\prime}+\lambda y=0$. As above, the weight function $p(x)$ for the operator

$$
L(\gamma)=\left(1-x^{2}\right) y^{\prime \prime}+(\alpha x+\beta) y^{\prime}
$$

is

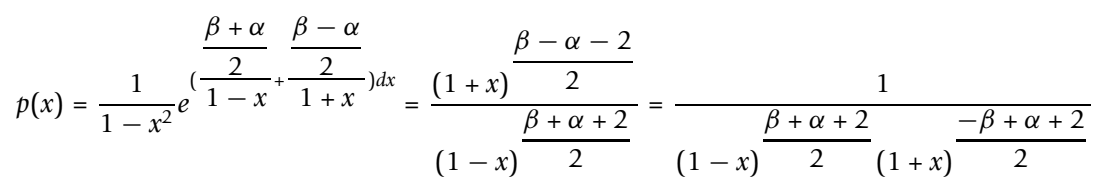

So $\int_{-1}^{1} p(x) f(x) d x$ would be finite if $\beta+\alpha<0$ and $-\beta+\alpha<0$, that is, if $\alpha<\beta<-\alpha$.

The weight is not differentiable at the end points of the interval. So, first consider $L$ operating on twice differentiable functions on the interval $[-1+\varepsilon, 1-\varepsilon]$. If $u, v$ are functions in this class then by Proposition 2.5,

$$
\begin{aligned}
\int_{-1+\varepsilon}^{1-\varepsilon} p(x) L(u(x)) v(x) d x & -\int_{-1+\varepsilon}^{1-\varepsilon} p(x) u(x) L(v(x)) d x \\
& =\left.p(x) a(x)\left(u(x) v^{\prime}(x)-u^{\prime}(x) v(x)\right)\right|_{-1+\varepsilon} ^{1-\varepsilon}
\end{aligned}
$$

Moreover, $\left(1-x^{2}\right) p(x)=(1-x) \frac{-(\beta+\alpha)}{2}(1+x) \frac{\beta-\alpha}{2}$ is continuous on the interval $[-1,1]$ and vanishes at the end-points -1 and 1 . Therefore, if we define $(u, v)=\lim _{\varepsilon \rightarrow 0} \int_{-1+\varepsilon}^{1-\varepsilon} p(x) u(x) v(x) d x$ then $L$ would be a self-adjoint operator on all polynomials of degree $n$ and so, there must be, up to a scalar, a unique polynomial which is an eigenfunction of $L$ for eigenvalue $-n(n-1)+n \alpha$.

So, these polynomials satisfy the equation

$$
\left(1-x^{2}\right) y^{\prime \prime}+(\alpha x+\beta) y^{\prime}+(n(n-1)-n \alpha) y=0
$$

and this equation has unique monic polynomial eigenfunctions of every degree, which are all orthogonal.

4.2 The equation $t(1-t) y^{\prime \prime}+(1-t) y+\lambda y=0$

This equation is investigated in [4] and the eigenvalues determined experimentally, by machine computations. Here, we will determine the eigenvalues in the framework provided by Propositions 2.1 and 2.5 .

Let $L(y)=t(1-t) y^{\prime \prime}+(1-t) y^{\prime}$. Let $\mathbb{P}_{n}$ be the space of al polynomials of degree at most $n$. As $L$ maps $\mathbb{P}_{n}$ into itself, the eigenvalues of $L$ are given by the coefficient of $x^{n}$ in $L\left(x^{n}\right)$. The eigenvalues turn out to be $-n^{2}$. As these eigenvalues are distinct, there is, up to a constant, a unique polynomial of degree $n$ which is an eigenfunction of $L$.

The weight function is $p(t)=\frac{1}{|1-t|}=\frac{1}{1-t}$ on the interval $[0,1]$ and it is not integrable. However, as $L(y)(1)=0$, the operator maps the space $V$ of all polynomials that are multiples of $(1-t)$ into itself. Moreover, $\int_{0}^{1} p(t)((1-t) \psi(t))^{2} d t$ is finite. 
The requirement for $L$ to be self-adjoint on $V$ is $\left.t\left(\xi \eta^{\prime}-\xi^{\prime} \eta\right)\right|_{0} ^{1}=0$ for all $\xi, \eta$ in $V$. As $\xi, \eta$ vanish at 1 , the operator $L$ is indeed self-adjoint on $V$.

Let $V_{n}=(1-t) \mathbb{P}_{n}$, where $\mathbb{P}_{n}$ is the space of all polynomials of degree at most $n$. As the codimension of $V_{n}$ in $V_{n+1}$ is 1 , the operator $L$ must have an eigenvector in $V_{n}$ for all the degrees from 1 to $n+1$. If $y=(1-t) \psi$ is an eigenfunction and $\operatorname{deg}(\psi)=n$, then, by the argument as in the examples above, we see that the corresponding eigenvalue is $\lambda=-(n+1)^{2}$.

Therefore, up to a scalar, there is a unique eigenfunction of degree $n+1$ which is a multiple of $1-t$ and all these functions are orthogonal for the weight $p(t)=\frac{1}{1-t}$.

Using the uniqueness up to scalars of these functions, the eigenfunctions are determined by the differential equation and can be computed explicitly.

\subsection{Romanovski polynomials}

These polynomials are investigated in [5,7] and their finite orthogonality is also proved there. Here, we establish this in the framework of Proposition 2.5.

The Romanovski polynomials are eigenfunctions of the operator $L(y)=\left(1+x^{2}\right) y^{\prime \prime}+$ $(\alpha x+\beta) y^{\prime}$. For $\alpha>0$, or $\alpha<0$, $\alpha$ not an integer, there is only one monic polynomial in every degree which is an eigenfunction of $L$; for $\alpha$ a non-positive integer, the eigenspaces can be two-dimensional for certain values of $\beta$ (Proposition 2.3).

The formal weight function is $p(x)=\left(x^{2}+1\right)^{\left(\frac{\alpha-2}{2}\right)} e^{\beta \tan ^{-1}(x)}=\left(x^{2}+1\right)^{\frac{\gamma}{2}} e^{\beta \tan ^{-1}(x)}$, where $\gamma=\alpha-2$. Therefore, a polynomial of degree $N$ is integrable over the reals with weight $p$ if and only if $N+\gamma+1<0$; and if the product of two polynomials $P, Q$ is integrable, then the polynomials are themselves integrable for the weight $p$.

As in Proposition 2.5, we find that $(L P, Q)-(P, L Q)=\left.\left(x^{2}+1\right) p(x)\left(P Q^{\prime}-P^{\prime} Q\right)\right|_{-\infty} ^{\infty}$, because if $\operatorname{deg}(P) \neq \operatorname{deg}(Q)$ then the product $\left(x^{2}+1\right) p(x)\left(P Q^{\prime}-P^{\prime} Q\right)$ is asymptotic to $x^{2+\gamma+\operatorname{deg}(P)+\operatorname{deg}(Q)-1}=x^{\operatorname{deg}(P)+\operatorname{deg}(Q)+\gamma+1}$ and $\operatorname{deg}(P)+\operatorname{deg}(Q)+\gamma+1<0$.

Therefore, if $P, Q$ are integrable eigenfunctions of $L$ with different eigenvalues and $\operatorname{deg}(P)+\operatorname{deg}(Q)+\gamma+1)<0$, then $P, Q$ are orthogonal.

For several non-trivial applications to problems in Physics, the readers are referred to [5].

\section{Acknowledgements}

The authors thank the referees for their valuable comments and Professor A. W. Knapp for his comments and suggestions on the first version of this article. They also thank the King Fahd University of Petroleum and Minerals for its support and excellent research facilities.

Authors' contributions

This was a joint work in every aspect. All the authors have read and approved the final manuscript.

Competing interests

The authors declare that they have no competing interests.

Received: 25 September 2011 Accepted: 28 November 2011 Published: 28 November 2011

References

1. Routh, EJ: On some properties of certain solutions of a differential equation of second order. Proc Lond Math Soc. 16 245-261 (1884). doi:10.1112/plms/s1-16.1.245

2. Bochner, S: Uber Sturm-Liouvillesche Polynomsysteme. Math Z. 29, 730-736 (1929). doi:10.1007/BF01180560

3. Brenke, WC: On polynomial solutions of a class of linear differential equations of the second order. Bull A M S. 36, 77-84 (1930). doi:10.1090/S0002-9904-1930-04888-0 
4. Chaudhry, MA, Qadir, A: Identity-type functions and polynomials. Int J Math Math Sci. 2007(94204), 21-31 (2007)

5. Raposo, AP, Weber, HJ, Alvarez-Castillo, D, Kirchbach, M: Romanovski polynomials in selected physics problems. Cent Eur J Phys. 5(3), 253-284 (2007). doi:10.2478/s11534-007-0018-5

6. Ismail, M: Classical and Quantum Orthogonal Polynomials in One Variable. Cambridge University Press (2005)

7. Weber, HJ: Connections between real polynomial solutions of hypergeometric-type differential equations with Rodrigues formula. Cent Eur J Math. 5(2), 415-427 (2007). doi:10.2478/s11533-007-0004-6

8. Birkhoff, G, Rota, GC: Ordinary Differential Equations. John Wiley and Sons, NY, 3 (1978)

9. Hilbert, D, Courant, R: Methods of Mathematical Physics. Interscience Publishers, NY (1953)

10. Alvarez-Castillo, DE: Exactly solvable potentials and Romanovski polynomials in quantum mechanics. Thesis, Institute of Physics at the Autonomous University of San Luis Potosi, Mexico rXiv (2007). 0808.1642

doi:10.1186/1687-1847-2011-58

Cite this article as: Azad et al: Polynomial solutions of differential equations. Advances in Difference Equations 2011 2011:58.

Submit your manuscript to a SpringerOpen ${ }^{\odot}$ journal and benefit from:

$\rightarrow$ Convenient online submission

- Rigorous peer review

- Immediate publication on acceptance

- Open access: articles freely available online

- High visibility within the field

- Retaining the copyright to your article

Submit your next manuscript at $\gg$ springeropen.com 